\title{
A simple model for rapidity fluctuations in the initial state of ultra-relativistic heavy-ion collisions
}

\author{
Wojciech Broniowski ${ }^{1,2, \text { f }}$ and Piotr Bożek ${ }^{3, \dagger}$ \\ ${ }^{1}$ The H. Niewodniczański Institute of Nuclear Physics, \\ Polish Academy of Sciences, 31-342 Cracow, Poland \\ ${ }^{2}$ Institute of Physics, Jan Kochanowski University, 25-406 Kielce, Poland \\ ${ }^{3}$ AGH University of Science and Technology, Faculty of Physics and \\ Applied Computer Science, al. Mickiewicza 30, 30-059 Cracow, Poland
}

(Dated: 30 November 2015)

\begin{abstract}
Two-particle pseudorapidity correlations are analyzed in a simple model, where in the initial stage of the reaction multiple sources, extended in rapidity, are created. We show how the fluctuations of the length of the sources in rapidity generate correlations in the initial entropy deposition, which later contribute to the observed longitudinal correlations in hadron production. Our analysis, which is analytic and leads to straightforward formulas, allows us to understand the structure of the correlations, in particular to identify the component related to the fluctuation of the numbers of sources and the component from the length fluctuations. We also present the results in terms of the expansion in the basis of the Legendre polynomials. A number of further effects is discussed, such as smearing of the pseudorapidity distributions or resonance decays. Our results reproduce qualitatively and semiquantitatively the basic features of the recent measurements at the LHC.
\end{abstract}

PACS numbers: $25.75 .-\mathrm{q}, 25.75 \mathrm{Gz}, 25.75 . \mathrm{Ld}$

\section{INTRODUCTION}

Recently, new data for the pseudorapidity correlations in $\mathrm{Pb}-\mathrm{Pb}, \mathrm{p}-\mathrm{Pb}$, and $\mathrm{p}-\mathrm{p}$ collisions at the Large Hadron Collider energies were released by the ATLAS Collaboration [1, 2]. These studies continue the longstanding experimental [3] and theoretical [4,8] efforts aimed at understanding of this important phenomenon, sensitive to the dynamics of the collision in its earliest stages.

This paper is devoted to theoretical understanding of the newest results [1, 2. Our study is based on what we call the longitudinally-extended source model. The initial entropy distribution in the longitudinal direction originates from the decay of strings or flux tubes connected to excited charges in the two colliding nuclei. The charges can be thought of as the wounded nucleons [9] or wounded quarks [10, thus are associated (attached) to a given colliding nucleus (cf. Fig. 1). A crucial aspect of the model is that the longitudinal position of the other end-point of the source is random, uniformly distributed in the central rapidity range. The use of the uniform distribution is a simplification, justified for the relatively narrow interval where the correlations are presently measured (pseudorapidity range $[-2.4,2.4]$ in the ATLAS experiment [1, 2]). Moreover, it allows for a straightforward analytic evaluation of the two-particle correlation function. This simple model grasps the essential features of realistic Monte Carlo models implementing the QCD string decays in the first stage of the collisions [11. The idea that entropy deposition originates from string-like

\footnotetext{
* Wojciech.Broniowski@ifj.edu.pl

$\dagger$ Piotr.Bozek@fis.agh.edu.pl
}

objects whose other end-point is randomly distributed in pseudorapidity $\eta$ is related to the Brodsky-GugnonKuhn mechanism [12]). It has also been discussed in the description of the fragmentation region in high energy collisions [13].

The study of multiplicity correlations can be conveniently done by projecting the two-particle correlations function on a two-dimensional basis of orthogonal polynomials [4. This method has been applied to $\mathrm{p}-\mathrm{p}, \mathrm{p}-\mathrm{Pb}$, and $\mathrm{Pb}-\mathrm{Pb}$ collisions by the ATLAS Collaboration [2] and was the subject of theoretical studies [4, 7, 8, 14, 16.

The analytic formulas obtained for the two-particle correlation functions allow us to understand the structure of the correlation function and to identify various components. As noticed in Ref. 4, the fluctuations of the numbers of sources alone generate non-trivial longitudinal correlations. We show, however, that the dominant effect comes from the correlation due to the emission of two particles from the same source, which is caused by the length fluctuations. This effect leads to the pair emission probability with a relatively long range in rapidity.

We find generically that the magnitude of the coefficients of the projection of the two-particle correlation functions on orthogonal polynomials is inversely proportional to the number of sources. With a natural proportionality relation between the number of sources and the final multiplicity, it can explain the experimentally observed scaling of these coefficients with the inverse number of observed hadrons, and the universality in A-A, p-A, and p-p collisions [2].

We recall that the presence of the long-range fluctuations of the entropy density in the longitudinal direction is also visible in the experimental data for the eventplane decorrelation in pseudorapidity [17]. The mechanism of the flow angle decorrelation has been discussed in 


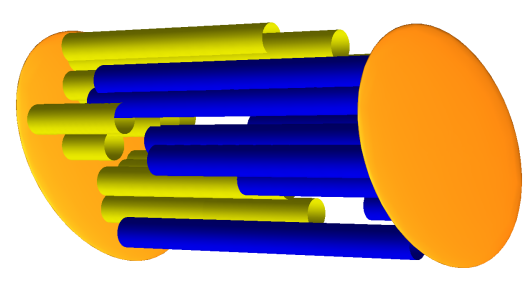

FIG. 1. Sources of fluctuating length, extending along the spatial pseudorapidity. The cartoon shows the situation in the early stage, right after the collision.

a number of papers 18 24. Fluctuations in the entropy deposition at different space-time rapidities lead to a relative torque of the elliptic and triangular flow angles at forward and backward rapidities.. The fluctuating endpoint mechanism discussed here has been applied numerically in Ref. 24 to describe the event-plane decorrelation (the torque effect [18) reported by the CMS Collaboration [17, where it was essential to describe the $\mathrm{p}-\mathrm{Pb}$ data. A conceptually similar model for the formation of the initial state for the hydrodynamic evolution, based on wounded quarks, was introduced in Ref. [8].

\section{EXPERIMENTAL MEASURES}

The preliminary experimental results of Ref. [1, 2] refer to correlations of two different charged hadrons of pseudorapidities $\eta_{1}$ and $\eta_{2}$, measured in a given centrality class. The relevant quantity is defined as

$$
C\left(\eta_{1}, \eta_{2}\right)=\frac{\left\langle\rho\left(\eta_{1}, \eta_{2}\right)\right\rangle}{\left\langle\rho\left(\eta_{1}\right)\right\rangle\left\langle\rho\left(\eta_{2}\right)\right\rangle}=\frac{S\left(\eta_{1}, \eta_{2}\right)}{B\left(\eta_{1}, \eta_{2}\right)},
$$

where $\rho\left(\eta_{1}, \eta_{2}\right)$ and $\rho\left(\eta_{1,2}\right)$ are the distributions of pairs and single particles, with $\langle$.$\rangle denoting the averages over$ events. In the experiment, the quantity is estimated by taking the ratio of the signal histogram $S$ with physical pairs over the histogram $B$ formed of mixed-event pairs. The ATLAS Collaboration [1] uses a measure obtained from $C\left(\eta_{1}, \eta_{2}\right)$ by dividing it by its marginal distributions, namely

$$
C_{N}\left(\eta_{1}, \eta_{2}\right)=\frac{C\left(\eta_{1}, \eta_{2}\right)}{C_{p}\left(\eta_{1}\right) C_{p}\left(\eta_{2}\right)}
$$

with

$$
C_{p}\left(\eta_{1}\right)=\int_{-Y}^{Y} d \eta_{2} C\left(\eta_{1}, \eta_{2}\right), \quad C_{p}\left(\eta_{2}\right)=\int_{-Y}^{Y} d \eta_{1} C\left(\eta_{1}, \eta_{2}\right)
$$

where $[-Y, Y]$ is the acceptance range for pseudorapidities $\eta_{1,2}$. In the ATLAS setup $Y=2.4$. This transformation reduces the effects of the overall multiplicity fluctuations on the shape of the correlation function. In ad- dition, the correlation functions are conventionally normalized to 1 , i.e., one introduces

$$
\bar{C}\left(\eta_{1}, \eta_{2}\right)=\frac{C\left(\eta_{1}, \eta_{2}\right)}{\int_{-Y}^{Y} d \eta_{1} \int_{-Y}^{Y} d \eta_{2} C\left(\eta_{1}, \eta_{2}\right)}
$$

and similarly $\bar{C}_{N}\left(\eta_{1}, \eta_{2}\right)$.

The $a_{n m}$ coefficients are defined via the expansion of the correlation functions in a basis of orthonormal functions [4]:

$$
\begin{aligned}
& a_{n m}=\int_{-Y}^{Y} \frac{d \eta_{1}}{Y} \int_{-Y}^{Y} \frac{d \eta_{2}}{Y} \bar{C}\left(\eta_{1}, \eta_{2}\right) T_{n}\left(\frac{\eta_{1}}{Y}\right) T_{m}\left(\frac{\eta_{1}}{Y}\right), \\
& a_{n m}^{N}=\int_{-Y}^{Y} \frac{d \eta_{1}}{Y} \int_{-Y}^{Y} \frac{d \eta_{2}}{Y} \bar{C}_{N}\left(\eta_{1}, \eta_{2}\right) T_{n}\left(\frac{\eta_{1}}{Y}\right) T_{m}\left(\frac{\eta_{1}}{Y}\right) .
\end{aligned}
$$

The choice made in Ref. 1, 2, 7, is

$$
T_{n}(x)=\sqrt{\frac{2 n+1}{2}} P_{n}(x),
$$

where $P_{n}(x)$ are the Legendre polynomials. The normalization is such that the functions $T_{n}(x)$ satisfy the orthonormality condition $\int_{-1}^{1} d x T_{n}(x) T_{m}(x)=\delta_{n m}$.

\section{CORRELATION FUNCTION IN THE LONGITUDINALLY-EXTENDED SOURCE MODEL}

In a wide class of approaches, the initial stage of the ultra-relativistic $A+B$ reaction comprises individual collisions between nucleons or their constituents. As a result, local deposition of entropy takes place. In our analysis we assume that these sources of entropy may be associated with the $A$ or $B$ nuclei. The prototype is the wounded nucleon model [9, 25, 26, but one may also think of wounded constituent quarks [10, 27, 28] or diquarks [29]. The approach was successful in describing the transverse dynamics, were the distribution of the initial sources in the transverse plane at mid-rapidity was relevant [30.

For the multiplicity distributions, the considered approach can be applied both to elementary collisions, where no substantial final state interaction occurs, and to nuclear collisions, where particle production happens after a collective expansion stage. In the former case, the source distribution in rapidity are directly related to probabilities of particle production at a given rapidity, with a possible shift in rapidity at hadronization. In the latter case, the rapidity distribution from elementary sources forms the initial condition for the subsequent dynamical evolution of the system. The spacetime dynamics of high-energy collisions is expected to follow the inside-outside cascade [31, with particle production occurring around a common longitudinal proper time $\sqrt{t^{2}-z^{2}}$. Particles with rapidity $y$ are produced approximately at the space-time rapidity $\frac{1}{2} \log \left(\frac{t+z}{t-z}\right) \simeq y$. 
If an intermediate collective evolution of matter occurs, a similar argument applies for the initial conditions of the fluid, where the Bjorken scaling flow may be assumed. Subsequent longitudinal and transverse evolution of the medium influences strongly the transverse momentum spectra. The longitudinal dynamics is less pronounced, especially if the longitudinal pressure is reduced due to non-equilibrium corrections [32, 33.

In the following, we study the initial conditions in space-time rapidity, assuming that they are close to the final rapidity distribution of matter just before hadronization (or thermal hadron emission from the fluid). Moreover, we assume that the observed pseudorapidity distributions are close to the rapidity distribution of the emitting sources. The effect of smearing of the distribution in the process of hadronization or thermal hadron emission, followed with resonance decays, is estimated explicitly and found to reduce the correlations.

For the present goal of describing the longitudinal correlations, one needs sources that extend along the space-time rapidity. One may think here of strings or flux-tubes, which are pulled by a nucleon or constituent from nucleus $A$ or $B$. The model is depicted in Fig. 1. where the longitudinally-extended sources have fluctuating length. Our approach includes the following components:

1. Each source may be associated with a nucleon (or its constituent) belonging to nucleus $A$ or $B$. In a given collision we have $N_{A}$ sources associated to $A$ and $N_{B}$ sources associated to $B$. We introduce the short-hand notation

$$
N_{+}=N_{A}+N_{B}, \quad N_{-}=N_{A}-N_{B}
$$

for the total number of sources and their difference between $A$ and $B$.

2. The entropy deposition (particle emission) from different sources is independent from one another.

3 . In the rapidity range between the end-points, the entropy deposition from a given source is uniformly distributed in rapidity.

4. The end-point of the longitudinally-extended source is randomly fluctuating, with the distribution adjusted in such a way that the phenomenological [34] average distribution from a source is reproduced. In the mid-rapidity region it leads to a uniform distribution of the end-point in space-time rapidity (cf. Appendix B).

5. In addition, we may overlay a distribution of strength over the sources 35, described by the random variable $\omega$. A statistical measure that appears in our formulas is

$$
s(\omega)=\frac{\operatorname{var}(\omega)}{\langle\omega\rangle^{2}} .
$$

6. As mentioned before, the role of a possible intermediate evolution stage (hydrodynamics, transport) is solely to provide an event-by-event mapping between the original distribution of entropy in spatial rapidity and the final distribution of hadrons in pseudorapidity.

7. In the late stage, we also discuss the effects of hadronization or thermal hadron emission and resonance decays.

The details of the derivation of the correlation function $C\left(\eta_{1}, \eta_{2}\right)$ are given in Appendices A C. Since we compare the results for the model with and without the length fluctuations, we introduce a parameter $r$ into our formulas, with $r=1$ and $r=0$ corresponding to present or absent length fluctuations, respectively. We also introduce the short-hand notation

$$
u_{1,2}=\eta_{1,2} / y_{b},
$$

with $y_{b}$ denoting a parameter close to the rapidity of the beam (cf. Appendix B). We have the following analytic expression for the correlation function (1) in the midrapidity region:

$$
\begin{aligned}
C\left(\eta_{1}, \eta_{2}\right)=1 & +\frac{1}{\left[\left\langle N_{+}\right\rangle+\left\langle N_{-}\right\rangle u_{1}\right]\left[\left\langle N_{+}\right\rangle+\left\langle N_{-}\right\rangle u_{2}\right]} \\
& \times\left\{\left\langle N_{+}\right\rangle\left[r\left(1-u_{1} u_{2}-\left|u_{1}-u_{2}\right|\right)+s(\omega)\left(1+r+(1-r) u_{1} u_{2}-r\left|u_{1}-u_{2}\right|\right)\right]+\right. \\
& \left.+\left\langle N_{-}\right\rangle s(\omega)\left(u_{1}+u_{2}\right)+\operatorname{var}\left(N_{+}\right)+\operatorname{var}\left(N_{-}\right) u_{1} u_{2}+\operatorname{cov}\left(N_{+}, N_{-}\right)\left(u_{1}+u_{2}\right)\right\},
\end{aligned}
$$

where the statistical moments are evaluated over the events. We note the obvious symmetry $\eta_{1} \leftrightarrow \eta_{2}$ (particles are not distinguishable).

The correlations originate from three kinds of effects:

1. the event-by-event fluctuation of the number of sources (the last three terms in curly brackets),

2. the overlaid fluctuation of strength of sources (the terms including $s(\omega)$,

3. and the length fluctuations (entering when $r=1$ ). 
The following structures appear: $u_{1} u_{2}, u_{1}+u_{2}$ (only for asymmetric collisions $A \neq B$ ), and $\left|u_{1}-u_{2}\right|$ (only when the length fluctuations are present).

For the symmetric case $A=B$ Eq. 10 simplifies into

$$
\begin{aligned}
C\left(\eta_{1}, \eta_{2}\right) & =1+\frac{1}{\left\langle N_{+}\right\rangle^{2}} \\
& \times\left\{\left\langle N_{+}\right\rangle\left[r\left(1-u_{1} u_{2}-\left|u_{1}-u_{2}\right|\right)+s(\omega)\left(1+r+(1-r) u_{1} u_{2}-r\left|u_{1}-u_{2}\right|\right)\right]+\operatorname{var}\left(N_{+}\right)+\operatorname{var}\left(N_{-}\right) u_{1} u_{2}\right\},
\end{aligned}
$$

where we note the additional symmetry $\left(\eta_{1} \rightarrow-\eta_{1}, \eta_{2} \rightarrow-\eta_{2}\right)$. For the strongly asymmetric case $\left(N_{A} \gg N_{B}\right)$ we get

$$
C\left(\eta_{1}, \eta_{2}\right)=1+\frac{\operatorname{var}\left(N_{A}\right)}{\left\langle N_{A}\right\rangle^{2}}+\frac{s(\omega)}{\left\langle N_{A}\right\rangle}+r \frac{[1+s(\omega)]\left[1-u_{1} u_{2}-\left|u_{1}-u_{2}\right|\right]}{\left\langle N_{A}\right\rangle\left(1+u_{1}\right)\left(1+u_{2}\right)}+\mathcal{O}\left(N_{B} / N_{A}\right),
$$

In the limiting case of no length fluctuations $(r=0)$ and no overlaid distribution $(s(\omega)=0)$ for symmetric collisions, we recover from Eq. (11) the result by Bzdak and Teaney [4,

$$
C\left(\eta_{1}, \eta_{2}\right)=1+\frac{\operatorname{var}\left(N_{+}\right)+\operatorname{var}\left(N_{-}\right) u_{1} u_{2}}{\left\langle N_{+}\right\rangle^{2}},
$$

which demonstrates that the fluctuation of $N_{A}$ vs $N_{B}$ induces the $\eta_{1} \eta_{2}$ structure in $C\left(\eta_{1}, \eta_{2}\right)$.

We remark that in the Glauber model one has approximately $\operatorname{var}\left(N_{+}\right) \sim\left\langle N_{+}\right\rangle$and $\operatorname{var}\left(N_{-}\right) \sim\left\langle N_{+}\right\rangle$, hence Eq. (11) leads to the approximate $1 /\left\langle N_{+}\right\rangle$scaling of $C\left(\eta_{1}, \eta_{2}\right)-1$ for the symmetric case. Similarly, from Eq. (12) we get the scaling $1 /\left\langle N_{A}\right\rangle$ for the strongly asymmetric case.

\section{LONGITUDINAL CORRELATIONS IN THE WOUNDED NUCLEON MODEL}

To see the importance of the length fluctuations, we have carried out a GLISSANDO [36] simulation in the wounded nucleon model for $\mathrm{Pb}+\mathrm{Pb}$ collisions at $2.76 \mathrm{TeV}$. In this case $N_{A}$ and $N_{B}$ are the wounded nucleons in the $A$ and $B$ nuclei. The simulation is needed solely to obtain the statistical averages $\left\langle N_{+}\right\rangle, \operatorname{var}\left(N_{+}\right)$, and $\operatorname{var}\left(N_{-}\right)$, as the dependence on $\eta_{1}$ and $\eta_{2}$ is analytic.

Some explanation is needed concerning the overlaid distribution of $\omega$. The quantity $\int_{-Y}^{Y} d \eta_{1} \int_{-Y}^{Y} d \eta_{2} C\left(\eta_{1}, \eta_{2}\right)$ measures the overall multiplicity fluctuation in the experimental pseudorapidity coverage. Therefore, when comparing the models with and without length fluctuations we should make this quantity equal. This requirement yields, with the help of Eq. (11), the condition

$$
s(\omega)_{\text {length ff. }}=\frac{3 s(\omega)_{\text {no length fl. }}+2 \frac{Y}{y_{p}}-3}{6-2 \frac{Y}{y_{p}}} .
$$

This equation shows, according to the expectations, that without the length fluctuations we must add more fluctuations in $\omega$ to obtain the same amount of the overall multiplicity fluctuations. In Refs. [24, 35, we

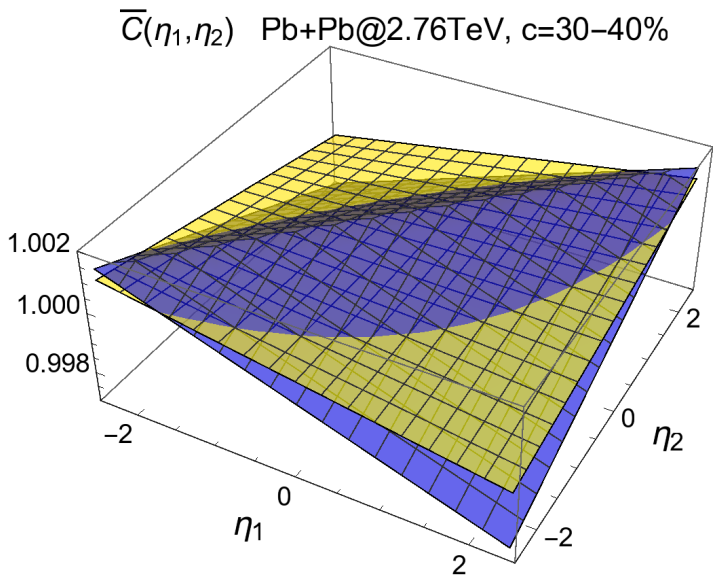

FIG. 2. (Color online) Correlation function $\bar{C}\left(\eta_{1}, \eta_{2}\right)$ for $\mathrm{Pb}-$ $\mathrm{Pb}$ collisions at $2.76 \mathrm{TeV}$ for centrality $c=30-40 \%$, obtained from Eqs. 4 11). The flat (light color) sheet corresponds to the case without the length fluctuations, whereas the sheet with the elongated maximum (darker color) corresponds to the model with the length fluctuations.

have checked that the overlaid $\Gamma$ distribution describes properly the multiplicity distribution in $\mathrm{p}-\mathrm{Pb}$ collisions at $5.02 \mathrm{TeV}$. The parameters for the model without the length fluctuations yield $s(\omega) \sim 1$, hence we take $s(\omega)_{\text {no length fl. }}=1$, and, correspondingly to Eq. 14, $s(\omega)_{\text {length fl. }}=Y /\left(3 y_{p}-Y\right) \simeq 0.1$. Such a low value for the model with the length fluctuations present is consistent with the observation made in Ref. [8, where no overlaid distribution was needed to reproduce the multiplicity spectra.

The result for the normalized correlation function (4) for $\mathrm{Pb}-\mathrm{Pb}$ collisions at $2.76 \mathrm{TeV}$ for a sample centrality $c=30-40 \%$ is shown in Fig. 2. We note a vivid difference between the models without and with the length fluctuations. Whereas the former case shows a rather flat structure, including the $\eta_{1} \eta_{2}$ term, the latter displays an elongated maximum, due to the $\left|\eta_{1}-\eta_{2}\right|$ structure resulting from the length fluctuations.

In Fig. 3 we show the corresponding function $\bar{C}_{N}\left(\eta_{1}, \eta_{2}\right)$. We note the generation of the ridge with 


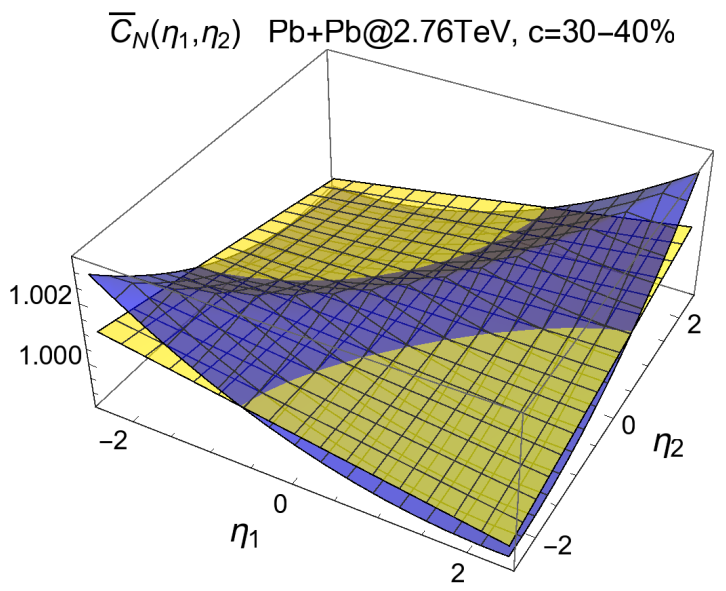

FIG. 3. (Color online) Same as Fig. 2 but for the function $\bar{C}_{N}\left(\eta_{1}, \eta_{2}\right)$.

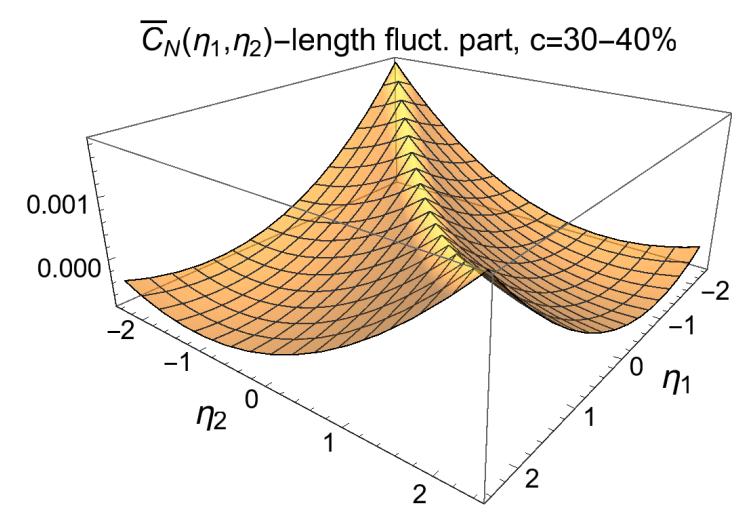

FIG. 4. (Color online) The difference of the correlation function from Fig. (3) with and without length fluctuations.

a saddle. The shape is simply caused by the definition (2); as the marginal distributions $C_{p}(\eta)$ have a maximum at $\eta=0$, the denominator in Eq. (2) relatively enhances the part of the plot at larger values of $\eta_{1}$ and $\eta_{2}$. Hence the generation of the saddle in the ridge (as seen in the experiment [1]) is natural from our expressions.

We note that the shape of the correlation functions with the length fluctuations displayed in Figs. 2 and 3 is qualitatively very similar to the experimental results, cf. Fig. 1 and 3 in Ref. 1]. Also, the values are comparable. At other centralities the shape of our correlation functions is as in Figs. 2 and 3 , but the size reflects the approximate $1 /\left\langle N_{+}\right\rangle$scaling.

To focus on the contribution from the length fluctuations, in Fig. 4 we display the difference of the cases including and excluding this effect. We note that the half-width of the ridge structure in the $\eta_{1}-\eta_{2}$ coordinate is about 2 units of pseudorapidity. The effect is clearly seen in Fig. 5 , where we show the sections of Fig. (3) along the line $\eta_{1}+\eta_{2}=0$.

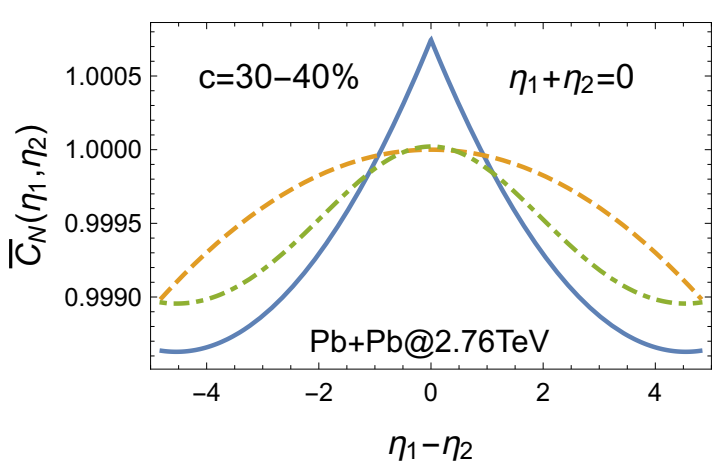

FIG. 5. (Color online) Sections of $\bar{C}_{N}\left(\eta_{1}, \eta_{2}\right)$ along the line $\eta_{1}+\eta_{2}=0$. The solid (dashed) lines correspond to the model with (without) the length fluctuations plotted in Fig. 3 The dot-dashed curve shows the result of smearing of the model with the length fluctuations with $\sigma_{\eta}=1$, as described in Sec. VI.

\section{THE $a_{n m}$ COEFFICIENTS}

Simple analytic expressions for the $a_{n m}$ coefficients for symmetric collisions may be obtained from Eq. (11). We find (for $n, m>0$ )

$$
\begin{aligned}
a_{n n} & =\frac{\frac{\operatorname{var}\left(N_{-}\right)}{\left\langle N_{+}\right\rangle}+(1-r) s(\omega)-r}{6\left\langle N_{+}\right\rangle} \frac{Y^{2}}{y_{b}^{2}} \delta_{n 1} \\
& +r \frac{s(\omega)+1}{(2 n-1)(2 n+3)\left\langle N_{+}\right\rangle} \frac{Y}{y_{b}}, \\
a_{n, n+2} & =a_{n+2, n}= \\
& =-r \frac{s(\omega)+1}{2(2 n+3) \sqrt{(2 n+1)(2 n+5)}\left\langle N_{+}\right\rangle} \frac{Y}{y_{b}},
\end{aligned}
$$

with all remaining combinations of $n$ and $m$ yielding $a_{n m}=0$. The first two terms in $a_{n n}$ originate from the $\eta_{1} \eta_{2}$ piece, while the other terms come from $\left|\eta_{1}-\eta_{2}\right|$. We note several facts:

1. In the model without length fluctuations $(r=0)$ we only have $a_{11} \neq 0$, which complies to Eq. (13).

2. The coefficients scale as $1 /\left\langle N_{+}\right\rangle$. For $a_{11}$ there may be slight departures from this scaling from the term $\operatorname{var}\left(N_{-}\right) /\left\langle N_{+}\right\rangle$, whereas for other coefficients the scaling is exact.

3. The coefficients drop with the value of the rank $n$, with the behavior $1 / n^{2}$ at large $n$.

Analogous analytic expressions for the $a_{n m}^{N}$ coefficients are lengthy, so we present them numerically. Their values are close to the $a_{n m}$ coefficients, with larger departure at low values of $\left\langle N_{+}\right\rangle$. This feature is seen for $a_{11}$ and $a_{11}^{N}$ from Fig. 6. We note that the difference between the model with and without length fluctuations is large (about a factor of 2).

From the occurrence of $\eta^{2}$ in Eq. C3 it is clear that in the symmetric case the coefficients $a_{n m}^{N}$ are no longer 


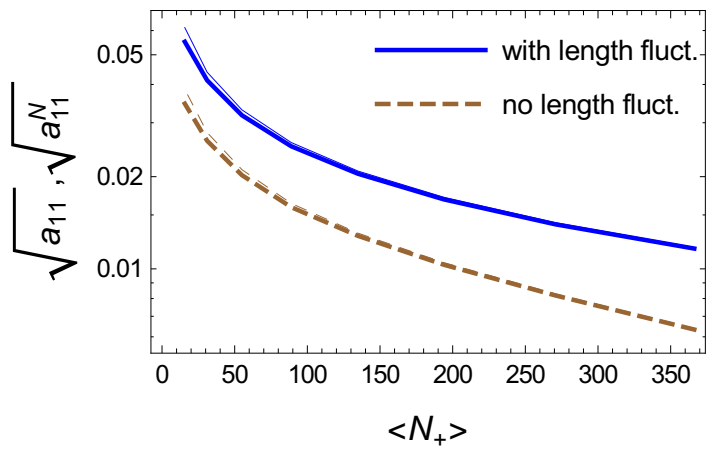

FIG. 6. (Color online) $\sqrt{a_{11}}$ and $\sqrt{a_{11}^{N}}$ (thin and thick lines, respectively) in the wounded nucleon model for $\mathrm{Pb}+\mathrm{Pb}$ collisions at $2.76 \mathrm{TeV}$.

limited to the tridiagonal structure of Eq. (15), but all combinations with $n$ and $m$ different by multiples of 2 are possible. However, the coefficients with $|n-m|>2$ are strongly suppressed with powers $1 /\left\langle N_{+}\right\rangle^{|m-n| / 2}$.

For the asymmetric case all combinations of $n$ and $m$ are possible, with a similar effect of suppression as one departs from the diagonal. For $a_{11}$ we find

$$
\begin{aligned}
a_{11}= & r(s(\omega)+1) y_{p} \times \\
& \frac{3 y_{p}\left(Y^{2}-y_{p}{ }^{2}\right) \operatorname{arctanh}\left(\frac{Y}{y_{p}}\right)+3 y_{p}{ }^{2} Y-2 Y^{3}}{2\left\langle N_{A}\right\rangle Y^{4}} \\
= & r \frac{(s(\omega)+1) Y}{5\left\langle N_{A}\right\rangle y_{p}}+r \frac{3(s(\omega)+1) Y^{3}}{35\left\langle N_{A}\right\rangle y_{p}{ }^{3}}+\mathcal{O}\left(Y^{4} / y_{p}^{4}\right) .
\end{aligned}
$$

\section{A. Universal scaling}

We notice that to leading order in $Y / y_{p}$, i.e., for small acceptance compared to the beam rapidity, we have for both the symmetric and asymmetric cases the universal behavior

$$
a_{11}=r \frac{(s(\omega)+1)}{5} \frac{Y}{y_{p}} \frac{1}{\langle N\rangle}+\ldots,
$$

where the dots indicate higher-order terms in $Y / y_{p}$. Here $N$ denotes $N_{+}$for the symmetric case, and $N_{A}$ for the asymmetric case, i.e., the total number of sources. Since for the ATLAS coverage $Y / y_{p} \sim 30 \%$, higher-order terms can be neglected and the universal (i.e., reactionindependent) $1 /\langle N\rangle$ scaling of Eq. (17), with the same prefactor, holds to a good accuracy.

In the scaling equation (17) the quantity $\langle N\rangle$ represents the average number of independent longitudinally extended sources (strings, flux tubes), emitting particles in the experimental acceptance window. In the illustrative calculation of the preceding section we have used wounded nucleons as sources. However, it should be noted that the same expressions apply if wounded quarks are connected to the decaying flux tubes. The ATLAS experiment finds the $1 / N_{\text {ch }}$ scaling for the $a_{n m}$ coefficients 2, where $N_{\text {ch }}$ denotes the number of observed

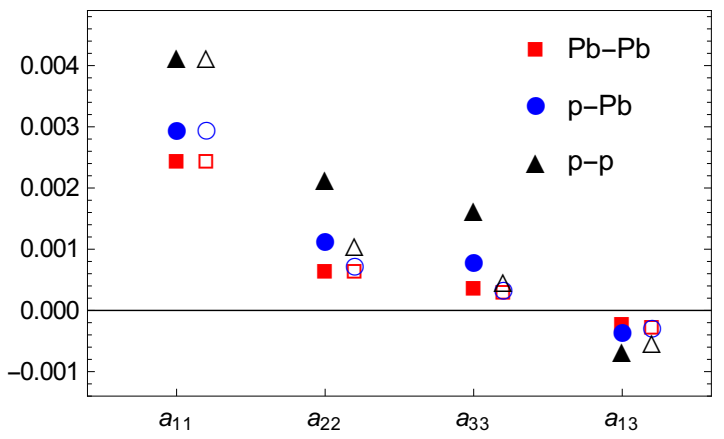

FIG. 7. (Color online) The $a_{n m}$ coefficients evaluated in the model (open symbols) and compared to the preliminary ATLAS data (filled symbols) for the same-charge hadron pairs, takes from Fig. 7 of Ref. [1 for $N_{\mathrm{ch}}=100-120$. The experimental values of $a_{11}$ were used to determine the proportionality between $\left\langle N_{\mathrm{ch}}\right\rangle$ and the average number of sources $\langle N\rangle$ for each reaction.

charged hadrons, with approximately the same prefactor for $\mathrm{Pb}-\mathrm{Pb}, \mathrm{p}-\mathrm{Pb}$, and $\mathrm{p}-\mathrm{p}$ collisions when the short-range correlations are removed (cf. Fig. 14 of Ref. 2). If the scaling between the number of sources and the multiplicity of charged particles is linear,

$$
N \sim N_{\mathrm{ch}},
$$

then the scaling $a_{n m} \propto 1 /\langle N\rangle$ is transformed into the scaling $a_{n m} \propto 1 /\left\langle N_{c h}\right\rangle$, exactly as observed in the experimental data.

We note that the proportionality $(18)$, with $N$ as the number of wounded quarks, was checked [37, 38] to hold well at the RHIC energies. On the other hand, this proportionality does not hold when $N$ stands for the number of wounded nucleons. Thus we conclude that Eq. (17) together with the experimental $1 / N_{\text {ch }}$ scaling for the $a_{n m}$ coefficients conforms to the wounded quark picture of the high-energy nuclear reactions.

\section{B. Model vs data}

We now pass to comparing our model results to the data. Since the later ATLAS analysis 2 removes the short-distance component from the correlation function with a rather involved procedure, difficult to repeat in a model calculation, we resort to the preliminary data from Ref. 1 for the pair of particles of the same charge. These correlation functions are not strongly sensitive to resonance decays.

We can use the experimental values for the $a_{11}$ coefficients to fix the proportionality constant in Eq. (18). To reproduce the data for $a_{11}$ in Fig. 7 we have assumed that $\left\langle N_{\mathrm{ch}}\right\rangle=4.7\left\langle N_{+}\right\rangle$for $\mathrm{Pb}-\mathrm{Pb}$ collisions at $2.76 \mathrm{TeV}$, $\left\langle N_{\mathrm{ch}}\right\rangle=5.1\left\langle N_{A}\right\rangle$ for p-Pb collisions at $5.02 \mathrm{TeV}$, and $\left\langle N_{\mathrm{ch}}\right\rangle=8.1\left\langle N_{+}\right\rangle$for p-p collisions at $13 \mathrm{TeV}$. These coefficients have the interpretation of the average number of observed charged hadrons per source, and have 
reasonable values. They roughly reflect the growth of the multiplicity in p-p collisions with energy. Moreover, it should be noted that the same multiplicity classes in different collision systems correspond to a different percentile of the inelastic cross section. In high multiplicity p-p events one triggers on events with a higher multiplicity of hadrons per source. A detailed modeling of the multiplicity classes would require a Monte Carlo modeling and additional assumptions concerning the distribution of sources and the number of charged particles per source. These issues are outside the scope of the analytic calculation presented in this paper.

We note from Fig. 7 that the higher-rank coefficients are predicted to be significantly smaller than $a_{11}$. Whereas the case of $\mathrm{Pb}-\mathrm{Pb}$ is well reproduced for higher $n$ and $m$, the splitting between $\mathrm{Pb}-\mathrm{Pb}$ and $\mathrm{p}-\mathrm{Pb}$ or $\mathrm{p}-\mathrm{p}$ is too small. The higher-order coefficients are expected to be more sensitive to the details of production mechanism that are not taken into account in our analytic calculation, such as a non-uniform distribution of flux-tube ends, matter evolution in rapidity, difference between rapidity and pseudorapidity, or hadronization effects. A strong sensitivity of higher order $a_{n m}$ coefficients to the details of the dynamics has been noticed in Ref. [8].

\section{FROM INITIAL STATE TO FINAL HADRONS}

Our analytic model uses several simplifying assumptions. We comment shortly about these issues and make an estimate of the most important effect.

The dynamics of particle creation acts differently in elementary collisions and in nuclear collisions. Hadronization in a small system is severely constrained by the local energy and charge conservation requirement [39], which leads to modifications of the correlation functions. Charge conservation generates a peak in the two-particle correlation as small pseudorapidity separations [40. The energy conservation in the string fragmentation mechanism gives a reduction of the emission probability for particles with similar rapidities. If a large collectively expanding source is created, the energy conservation effects are reduced. On the other hand, the longitudinal expansion of the fireball may induce a rescaling of the rapidity variable between the initial state and the matter at freeze-out. This would lead to some reduction of the correlation coefficients $a_{n m}$ in the final state as compared to the initial state.

The transformation from the density $\rho\left(\eta_{1}, \eta_{2}\right)$ into observed particles involves a hadronization mechanism. In elementary processes, it could be modeled as string decay or quark coalescence. In nuclear collisions, individual hadrons appear at freeze-out through thermal emission from fluid elements. In the first case, particle emission requires color and momentum exchanges with the rest of the system in order to fulfill the local conservation laws. In the case of the emission from a hot fireball, hadron

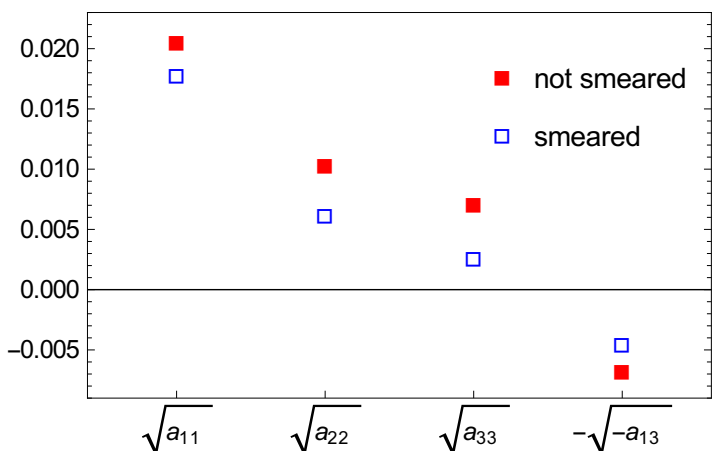

FIG. 8. (Color online) Square root of the $a_{n m}$ coefficients evaluated in the model without smearing (filled symbols) and with a Gaussian smearing of with $\sigma_{\eta}=1$ (empty symbols). $\mathrm{Pb}-\mathrm{Pb}$ collisions at $2.76 \mathrm{TeV}$, wounded nucleon model with GLISSANDO, centrality $c=30-40 \%$.

rapidities are washed out as compared to the fluid rapidity due to the thermal component in the momenta. A similar washing out of the distribution is expected from the decays of resonances.

Irrespective of the details of the mechanism of particle production, the pseudorapidity distribution of the observed particles is different than the two-particle density discussed so far. The effects can be approximately estimated as a convolution of the numerator of Eq. (1) with Gaussian form factors,

$$
S_{\mathrm{sm}}\left(\eta_{1}, \eta_{2}\right)=\int d \eta_{1}^{\prime} \int d \eta_{2}^{\prime} g\left(\eta_{1}, \eta_{1}^{\prime}\right) g\left(\eta_{2}, \eta_{2}^{\prime}\right) S\left(\eta_{1}^{\prime}, \eta_{2}^{\prime}\right)
$$

and similarly for the denominator $B\left(\eta_{1}, \eta_{2}\right)$. We take the following form of the smearing function

$$
g\left(\eta, \eta^{\prime}\right)=\frac{1}{\sqrt{2 \pi} \sigma_{\eta}} e^{-\frac{\left(\eta-\eta^{\prime}\right)^{2}}{2 \sigma_{\eta}^{2}}},
$$

with $\sigma_{\eta}=1$, which corresponds to a rather large smearing width of $\sqrt{2}$ for the difference $\eta_{1}-\eta_{2}$. We notice from the results shown in Fig. 8 that the smearing reduces the values of the $a_{n m}$ coefficients. As expected, the effect is stronger for higher-rank coefficients.

Finally, resonance decays are a significant source of two-particle correlations among produced hadrons of opposite charge. In Fig. 9 we recall the result of our analysis of Ref. 14] carried out in the wounded nucleon model without fluctuating ends. Comparing Fig. 9 to Fig. 3 , we note a significant relative size of the component from resonance decays.

\section{CONCLUSIONS}

The mechanism of energy deposition and particle creation in high-energy collisions in the longitudinal direction has been a subject of many recent studies. In this paper, we have investigated the two-particle correlations in 


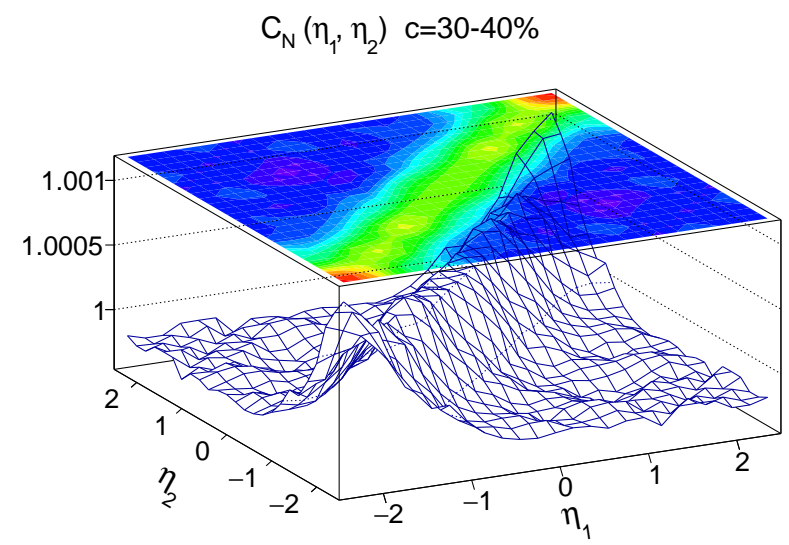

FIG. 9. (Color online) Contribution to the correlation function $C_{N}\left(\eta_{1}, \eta_{2}\right)$ from the resonance decays, reprinted from Fig. 2 of Ref. [14].

rapidity as measured by the ATLAS Collaboration [1, 2. A nice aspect of the proposed correlation measures is that they may be obtained analytically in a simple model based on independent longitudinally extended sources. Our model exemplifies the most important characteristics of a broad class of realistic models based on the decays of strings or flux tubes, while remaining simple enough to allow for the derivation of analytic formulas for the correlation function and for the coefficients of its expansion in orthogonal polynomials.

Our model of the initial energy (or entropy) deposition from flux tubes is a mechanism describing the formation of the initial state in the collisions, with the distribution involving two-particle correlations. The model has been shown previously to describe fairly well the decorrelation of the harmonic flow event-planes at different pseudorapidities, also in p- $\mathrm{Pb}$ collisions [24]. The correlations in the energy deposition from the early stage of the collision can be transmitted into final hadron distribution via different scenarios. In elementary collisions, the flux tubes decay directly into hadrons at hadronization, with subsequent resonance decays. In nuclear collisions (or, perhaps, highest-multiplicity p-p collisions), a fireball is formed that expands collectively and hadrons are emitted at freeze-out. To a good approximation, our analysis applies to both scenarios, as the hydrodynamic evolution of the fireball in the longitudinal direction for central rapidities is moderate.

The flux tubes are formed independently from one another from excited color charges in the target and the projectile. Our model explicitly reveals the two basic mechanism of generating fluctuations in rapidity: Firstly, an event-by-event asymmetry of the number of excited charges in the target and projectile leads to event-byevent fluctuations of the density and gives a correlation function of the form $C\left(\eta_{1}, \eta_{2}\right)=1+A \eta_{1} \eta_{2}+\ldots$, as already noticed by Bzdak and Teaney [4. The second mechanism originates from the random positions in rapidity of the end-points of the decaying sources (length fluctuations). We find that this effect generates the dominant contribution to the correlation function, roughly two times larger than the Bzdak-Teaney term. Both terms scale approximately as $1 /\langle N\rangle$, i.e., are inversely proportional to the number of sources. With the feature that the number of sources is proportional to the final multiplicity of charged hadrons, the model explains the experimental observation of a similar value of the $a_{11}$ coefficients in different systems at the same multiplicity [2, as well as its $1 /\left\langle N_{\mathrm{ch}}\right\rangle$ scaling,

Our analytic expressions for the correlation function and the $a_{n m}$ coefficients display the dependence on statistical measures of the sources and the overlaid distribution, on the assumed model of the end-point fluctuation, on the width of the experimental acceptance window in pseudorapidity, and on the indices $n$ and $m$. One should bear in mind that the derived formulas are modified by a number of omitted and potentially relevant effects, in particular hadronization and resonance decays. We have crudely estimated these effect by smearing the correlation function with Gaussian form factors, which leads to a sizable reduction of the $a_{n m}$ coefficients, stronger for larger $n$ and $m$. Our smearing procedure may be thought of as a way to remove some of the short-distance correlations. The resonance decays, analyzed via numerical simulations in Ref. 14, form a significant explicit contribution to the correlation function. It may be significantly reduced when the correlations of pairs of the same charge are considered.

\section{ACKNOWLEDGMENTS}

Research supported by the Polish Ministry of Science and Higher Education, (MNiSW), by the Polish National Science Centre grants DEC-2012/05/B/ST2/02528.

\section{Appendix A: Derivation of $C\left(\eta_{1}, \eta_{2}\right)$ in the longitudinally-extended source model}

The formula for the event-by-event averaged distribution of entropy is

$$
\begin{aligned}
& \left\langle\rho\left(\eta_{1} . \eta_{2}\right)\right\rangle= \\
& \quad=\left\langle N_{A}\right\rangle\left\langle f_{A}\left(\eta_{1}, \eta_{2}\right)\right\rangle+\left\langle N_{A}\left(N_{A}-1\right)\right\rangle\left\langle f_{A}\left(\eta_{1}\right)\right\rangle\left\langle f_{A}\left(\eta_{2}\right)\right\rangle \\
& \quad+\left\langle N_{B}\right\rangle\left\langle f_{B}\left(\eta_{1}, \eta_{2}\right)\right\rangle+\left\langle N_{B}\left(N_{B}-1\right)\right\rangle\left\langle f_{B}\left(\eta_{1}\right)\right\rangle\left\langle f_{B}\left(\eta_{2}\right)\right\rangle \\
& \quad+\left\langle N_{A} N_{B}\right\rangle\left[\left\langle f_{A}\left(\eta_{1}\right)\right\rangle\left\langle f_{B}\left(\eta_{2}\right)\right\rangle+\left\langle f_{B}\left(\eta_{1}\right)\right\rangle\left\langle f_{A}\left(\eta_{2}\right)\right\rangle\right],
\end{aligned}
$$

where the first term corresponds to the situation where both bins at $\eta_{1}$ and $\eta_{2}$ are fed from the production from the same source associated to nucleus $A$, the second term corresponds to production from two different sources belonging to $A$, the third and fourth term describe the analogous emission from the sources associated to $B$, finally, the last term corresponds to the emission from one source belonging to $A$ and the other to $B$. The 
functions $\left\langle f_{A, B}\left(\eta_{1}, \eta_{2}\right)\right\rangle$ and $\left\langle f_{A, B}\left(\eta_{1,2}\right)\right\rangle$ are the eventaveraged two-body and one-body emission profiles, constructed below.

With a simple rearrangement one may rewrite the above equation in the form

$$
\begin{aligned}
& \left\langle\rho\left(\eta_{1}, \eta_{2}\right)\right\rangle= \\
& \quad=\left\langle N_{A}\right\rangle \operatorname{cov}_{A}\left(\eta_{1}, \eta_{2}\right)+\left\langle N_{A}^{2}\right\rangle\left\langle f_{A}\left(\eta_{1}\right)\right\rangle\left\langle f_{A}\left(\eta_{2}\right)\right\rangle \\
& \quad+\left\langle N_{B}\right\rangle \operatorname{cov}_{B}\left(\eta_{1}, \eta_{2}\right)+\left\langle N_{B}^{2}\right\rangle\left\langle f_{B}\left(\eta_{1}\right)\right\rangle\left\langle f_{B}\left(\eta_{2}\right)\right\rangle \\
& \quad+\left\langle N_{A} N_{B}\right\rangle\left[\left\langle f_{A}\left(\eta_{1}\right)\right\rangle\left\langle f_{B}\left(\eta_{2}\right)\right\rangle+\left\langle f_{B}\left(\eta_{1}\right)\right\rangle\left\langle f_{A}\left(\eta_{2}\right)\right\rangle\right],
\end{aligned}
$$

where

$\operatorname{cov}_{i}\left(\eta_{1}, \eta_{2}\right)=\left\langle f_{i}\left(\eta_{1}, \eta_{2}\right)\right\rangle-\left\langle f_{i}\left(\eta_{1}\right)\right\rangle\left\langle f_{i}\left(\eta_{2}\right)\right\rangle, \quad i=A, B$.
For the event-by-event averaged one-body densities we simply have

$$
\langle\rho(\eta)\rangle=\left\langle N_{A}\right\rangle\left\langle f_{A}(\eta)\right\rangle+\left\langle N_{B}\right\rangle\left\langle f_{B}(\eta)\right\rangle .
$$

In the reference frame where the $A$ and $B$ nuclei move with equal and opposite velocities, we may decompose the emission profiles into parts symmetric and antisymmetric in spatial pseudorapidity.

$$
\begin{aligned}
& \left\langle f_{A}(\eta)\right\rangle=\left\langle f_{s}(\eta)\right\rangle+\left\langle f_{a}(\eta)\right\rangle,\left\langle f_{B}(\eta)\right\rangle=\left\langle f_{s}(\eta)\right\rangle-\left\langle f_{a}(\eta)\right\rangle, \\
& \left\langle f_{s}(\eta)\right\rangle=\left\langle f_{s}(-\eta)\right\rangle, \quad\left\langle f_{a}(\eta)\right\rangle=-\left\langle f_{a}(-\eta)\right\rangle .
\end{aligned}
$$

Then, after elementary transformations,

$$
\begin{aligned}
C\left(\eta_{1}, \eta_{2}\right)=1 & +\frac{1}{\left[\left\langle N_{+}\right\rangle\left\langle f_{s}\left(\eta_{1}\right)\right\rangle+\left\langle N_{-}\right\rangle\left\langle f_{a}\left(\eta_{1}\right)\right\rangle\right]\left[\left\langle N_{+}\right\rangle\left\langle f_{s}\left(\eta_{2}\right)\right\rangle+\left\langle N_{-}\right\rangle\left\langle f_{a}\left(\eta_{2}\right)\right\rangle\right]} \times \\
& \times\left\{\left\langle N_{A}\right\rangle \operatorname{cov}_{A}\left(\eta_{1}, \eta_{2}\right)+\left\langle N_{B}\right\rangle \operatorname{cov}_{B}\left(\eta_{1}, \eta_{2}\right)+\operatorname{var}\left(N_{+}\right)\left\langle f_{s}\left(\eta_{1}\right)\right\rangle\left\langle f_{s}\left(\eta_{2}\right)\right\rangle+\operatorname{var}\left(N_{-}\right)\left\langle f_{a}\left(\eta_{1}\right)\right\rangle\left\langle f_{a}\left(\eta_{2}\right)\right\rangle\right. \\
& \left.+\operatorname{cov}\left(N_{+}, N_{-}\right)\left[\left\langle f_{s}\left(\eta_{1}\right)\right\rangle\left\langle f_{a}\left(\eta_{2}\right)\right\rangle+\left\langle f_{a}\left(\eta_{1}\right)\right\rangle\left\langle f_{s}\left(\eta_{2}\right)\right\rangle\right]\right\},
\end{aligned}
$$

where $\operatorname{cov}\left(N_{+}, N_{-}\right)=\operatorname{var}\left(N_{A}\right)-\operatorname{var}\left(N_{B}\right)$. For the special case of symmetric collisions, where $\left\langle N_{A}\right\rangle=\left\langle N_{B}\right\rangle$ and $\operatorname{var}\left(N_{A}\right)=\operatorname{var}\left(N_{B}\right)$, Eq. A6 simplifies into

$$
\begin{aligned}
C\left(\eta_{1}, \eta_{2}\right) & =1+\frac{1}{\left\langle N_{+}\right\rangle^{2} f_{s}\left(\eta_{1}\right) f_{s}\left(\eta_{2}\right)} \times \\
& \times\left\{\left\langle N_{A}\right\rangle \operatorname{cov}_{A}\left(\eta_{1}, \eta_{2}\right)+\left\langle N_{B}\right\rangle \operatorname{cov}_{B}\left(\eta_{1}, \eta_{2}\right)+\operatorname{var}\left(N_{+}\right)\left\langle f_{s}\left(\eta_{1}\right)\right\rangle\left\langle f_{s}\left(\eta_{2}\right)\right\rangle+\operatorname{var}\left(N_{-}\right)\left\langle f_{a}\left(\eta_{1}\right)\right\rangle\left\langle f_{a}\left(\eta_{2}\right)\right\rangle\right\} .
\end{aligned}
$$

Another limiting case is for $N_{A} \gg N_{B}$, when

$$
C\left(\eta_{1}, \eta_{2}\right)=1+\frac{1}{\left\langle N_{A}\right\rangle^{2}\left\langle f_{A}\left(\eta_{1}\right)\right\rangle\left\langle f_{A}\left(\eta_{2}\right)\right\rangle}\left\{\left\langle N_{A}\right\rangle \operatorname{cov}_{A}\left(\eta_{1}, \eta_{2}\right)+\operatorname{var}\left(N_{A}\right)\left\langle f_{A}\left(\eta_{1}\right)\right\rangle\left\langle f_{A}\left(\eta_{2}\right)\right\rangle\right\}+\mathcal{O}\left(N_{B} / N_{A}\right) .
$$

A general remark may be made here. We note from Eq. A6 A8 that the correlations originate from two kinds of effects: the correlations from the emission off a single source (the terms with $\operatorname{cov}_{A, B}\left(\eta_{1}, \eta_{2}\right)$ ) and the fluctuations of the numbers of sources (the terms with variances). Therefore, as noticed already in Ref. 4, even if $\operatorname{cov}_{A, B}\left(\eta_{1}, \eta_{2}\right)=0$, we have a nontrivial correlation function $C\left(\eta_{1}, \eta_{2}\right)$.

\section{Appendix B: Fluctuating ends}

Białas-Czyż 34] have shown that the $\mathrm{d}+\mathrm{Au}$ collisions at RHIC may be understood within the wounded nucleon model if the average emission profiles have the form

$$
\left\langle f_{A}(\eta)\right\rangle=h(\eta) t\left(\eta ; y_{b}\right),\left\langle f_{B}(\eta)\right\rangle=h(\eta) t\left(-\eta ; y_{b}\right),
$$

where $y_{b}$ is a parameter of the order of the rapidity of the beam,

$$
t\left(\eta ; y_{b}\right)=\left\{\begin{array}{cl}
0 & \text { for } \eta<-y_{b} \\
\frac{y_{b}+\eta}{2 y_{b}} & \text { for }-y_{b} \leq \eta \leq y_{b} \\
1 & \text { for } y_{b}<\eta
\end{array}\right.
$$

and $h(\eta)$ is a suitable chosen function, symmetric in $\eta$, which turns out to be flat near the origin [41,

$$
h(\eta) \simeq \text { const } \text { for }-2.4<\eta<2.4
$$

at the LHC collision energies (as we work up to a multiplicative constant, we may take $h(\eta)=1$ ). Note the presence of the antisymmetric part in $t\left(\eta ; y_{b}\right)$ which describes the fact that the source from $A$ deposits entropy mostly forward, and the source from $B$ mostly backward in $\eta$. Parametrization (B1) has been later used in numerous papers [42] as a working model for the initial distributions.

We now step up in the model building and investigate models where the sources have randomly distributed 
ends. The constraint is that they must reproduce the phenomenologically successful Eq. (B1).

\section{Single-end fluctuations}

We consider first a simple model where the source has one end attached to the nucleus $A$ or $B$ (it is placed at pseudorapidity $y_{b}$ or $-y_{b}$, respectively), and the other end is fluctuating from source to source in the range $\left[-y_{b}, y_{b}\right]$. We assume a uniform production of entropy from a source with a random end-point $y$, i.e.,

$$
\begin{aligned}
& f_{A}(\eta ; y)=\theta\left(y<\eta<y_{b}\right), \\
& f_{B}(\eta ; y)=\theta\left(-y_{b}<\eta<y\right),
\end{aligned}
$$

where $\theta$ is a step function equal 1 when the argument is true, and 0 otherwise. The random end $y$ is generated according to a suitably chosen distribution $g(y)$. Averaging over events involves averaging over $y$, therefore

$$
\begin{aligned}
& \left\langle f_{A}(\eta)\right\rangle=\int_{-y_{b}}^{y_{b}} d y g(y) f_{A}(\eta ; y)=\int_{-y_{b}}^{\eta} d y g(y)=G(\eta)-G\left(-y_{b}\right), \\
& \left\langle f_{B}(\eta)\right\rangle=\int_{-y_{b}}^{y_{b}} d y g(y) f_{B}(\eta ; y)=\int_{\eta}^{y_{b}} d y g(y)=G\left(y_{b}\right)-G(\eta),
\end{aligned}
$$

with $G^{\prime}(y)=g(y)$. Therefore, to match to Eq. (B1) we must simply take for the length distribution function

$$
g(y)=d / d y\left\langle f_{A}(y)\right\rangle=-d / d y\left\langle f_{B}(y)\right\rangle .
$$

Since in the experimental coverage of the ATLAS experiment 2] $h(\eta)$ is flat within the range of Eq. (B3) (we may take $h(\eta)=1$ as normalization is not relevant), we find

$$
g(y)=\frac{1}{2 y_{b}},
$$

i..e., a uniform distribution of the end point, valid in the central pseudorapidity region.

The length fluctuations induce correlations, as we have

$$
\begin{aligned}
& \left\langle f_{A}\left(\eta_{1}, \eta_{2}\right)\right\rangle=\int_{-y_{b}}^{y_{b}} d y g(y) f_{A}\left(\eta_{1} ; y\right) f_{A}\left(\eta_{2} ; y\right) \\
& =\int_{-y_{b}}^{\min \left(\eta_{1}, \eta_{2}\right)} d y g(y)=\left\langle f_{A}\left(\min \left(\eta_{1}, \eta_{2}\right)\right)\right\rangle, \\
& \left\langle f_{B}\left(\eta_{1}, \eta_{2}\right)\right\rangle=\int_{-y_{b}}^{y_{b}} d y g(y) f_{B}\left(\eta_{1} ; y\right) f_{B}\left(\eta_{2} ; y\right) \\
& =\int_{\max \left(\eta_{1}, \eta_{2}\right)}^{y_{b}} d y g(y)=\left\langle f_{B}\left(\max \left(\eta_{1}, \eta_{2}\right)\right)\right\rangle .
\end{aligned}
$$

In the central region with Eq. B7] we have

$$
\begin{aligned}
& \left\langle f_{A}\left(\eta_{1}, \eta_{2}\right)\right\rangle=t\left[\min \left(\eta_{1}, \eta_{2}\right)\right], \\
& \left\langle f_{B}\left(\eta_{1}, \eta_{2}\right)\right\rangle=t\left[-\max \left(\eta_{1}, \eta_{2}\right)\right] .
\end{aligned}
$$

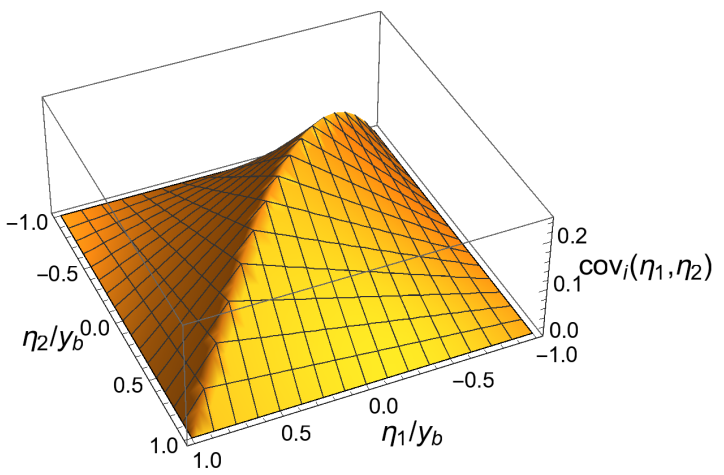

FIG. 10. (Color online) The covariance from single-end fluctuations, Eq. B10.

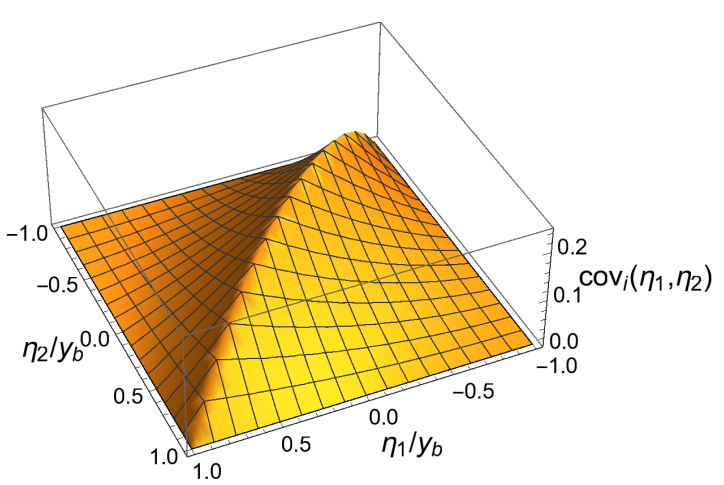

FIG. 11. (Color online) The covariance from double-end fluctuations, Eq. B15.

Consequently, we find

$$
\operatorname{cov}_{A}\left(\eta_{1}, \eta_{2}\right)=\operatorname{cov}_{B}\left(\eta_{1}, \eta_{2}\right)=\frac{y_{b}^{2}-\eta_{1} \eta_{2}-y_{b}\left|\eta_{1}-\eta_{2}\right|}{4 y_{b}^{2}}
$$

where we have used $\max (a, b)=(a+b+|a-b|) / 2$ and $\min (a, b)=(a+b-|a-b|) / 2$. Formula $\mathrm{B} 10)$ is illustrated in Fig. 10 .

Note that we have obtained correlation, although the emission was independent in the sense that we have used the product $f_{i}\left(\eta_{1} ; y\right) f_{i}\left(\eta_{2} ; y\right)$ in the integrand in Eq. (B8). The correlation is due to the common limit on emission of both particles from the fluctuating end-point $y$.

We also remark that the assumed formula (B2) simultaneously fixes the symmetric and antisymmetric parts in Eq. A5). In a more general situation this need not be the case, for instance we might have a larger symmetric component, replacing

$$
t\left(\eta ; y_{b}\right) \rightarrow t\left(\eta ; y_{b}\right)+\alpha,
$$

where $\alpha>0$. The presence of an extra symmetric component would reduce the correlations coming from the fluctuating end-points, since $f_{s}\left(\eta_{1}\right) f_{s}\left(\eta_{2}\right)$ appears in the denominator of $C\left(\eta_{1}, \eta_{2}\right)$. 


\section{Double-end fluctuations}

The derivation of the previous section may be straightforwardly extended to the case where both ends fluctuate. Then

$$
f_{A}\left(\eta ; y_{1}, y_{2}\right)=\theta\left(y_{1}<\eta<y_{2}\right)
$$

and

$$
\begin{aligned}
& \left\langle f_{A}(\eta)\right\rangle=\int_{-y_{b}}^{y_{b}} d y_{1} g_{1}\left(y_{1}\right) \int_{-y_{b}}^{y_{b}} d y_{2} g_{2}\left(y_{2}\right) f_{A}\left(\eta ; y_{1}, y_{2}\right) \\
& =\int_{-y_{b}}^{\eta} d y_{1} g_{1}\left(y_{1}\right) \int_{\eta}^{y_{b}} d y_{2} g_{2}\left(y_{2}\right) \\
& =\left[G_{1}(\eta)-G_{1}\left(-y_{b}\right)\right]\left[G_{2}\left(y_{b}\right)-G_{2}(\eta)\right]
\end{aligned}
$$

(similar expressions hold for $\left.\left\langle f_{B}(\eta)\right\rangle\right)$. In the present case, in general, we cannot uniquely obtain $G_{1}(y)$ and $G_{2}(y)$ by matching to $\left\langle f_{A}(y)\right\rangle$. However, in the case where one end of the source is close to the fragmentation region (as expected of wounded quarks, for example), its fluctuations do not enter the central region (B3) and effectively, for that domain, we get the model with single-end fluctuations of Sec. B 1 .

In the special case where both end-points fluctuate uniformly, we get from Eq, B13 the symmetric component

$$
f_{s}(\eta)=\frac{y_{b}^{2}-\eta^{2}}{4 y_{b}^{2}}
$$

as well as the contribution to the covariance

$$
\operatorname{cov}_{i}\left(\eta_{1}, \eta_{2}\right)=\frac{y_{b}^{2}-\min \left(\eta_{1}, \eta_{2}\right)^{2}}{4 y_{b}^{2}}-\frac{y_{b}^{2}-\eta_{1}^{2}}{4 y_{b}^{2}} \frac{y_{b}^{2}-\eta_{2}^{2}}{4 y_{b}^{2}}
$$

Equation (B15) is illustrated in Fig. 11. We note that the shape of the obtained covariance is qualitatively similar to the case of Fig. 10 , with the size smaller by $25 \%$, and a faster fall-off in $\left|\eta_{1}-\eta_{2}\right|$ for the case of double-end fluctuations.

\section{Appendix C: Fluctuating strength}

In Glauber-like models, a common ingredient is the overlaid distribution, i.e., on top of the sources we superpose a random distribution [36]. This describes the possibility that the sources may have randomly varying strength. The superposition is necessary to properly describe the multiplicity distributions of produced hadrons when the conventional Glauber model is used [35].
In our framework, for the model with single-end fluctuations, the fluctuating strength is incorporated as a generalization of Eq. (B4), namely

$$
\begin{aligned}
& f_{A}(\eta ; y)=\omega \theta\left(y<\eta<y_{b}\right), \\
& f_{B}(\eta ; y)=\omega \theta\left(-y_{b}<\eta<-y\right),
\end{aligned}
$$

where $\omega$ is a random variable giving the strength of the source. With these extra fluctuations we have the following generalization of Eqs. (B5 B10):

$$
\begin{aligned}
& \left\langle f_{A, B}(\eta)\right\rangle=\langle\omega\rangle t\left( \pm \eta ; y_{b}\right), \\
& \operatorname{cov}_{A, B}\left(\eta_{1}, \eta_{2}\right)=\left\langle\omega^{2}\right\rangle \frac{y_{b}^{2}-\eta_{1} \eta_{2}-y_{b}\left|\eta_{1}-\eta_{2}\right|}{4 y_{b}^{2}} \\
& +\operatorname{var}(\omega) \frac{y_{b}^{2}+\eta_{1} \eta_{2} \pm y_{b}\left(\eta_{1}+\eta_{2}\right)}{4 y_{b}^{2}} .
\end{aligned}
$$

The formulas for the correlation functions are given in Eq. 10, 12. For the marginal projections of Eq. (3) we find for the symmetric case

$$
\begin{aligned}
C_{p}(\eta) & =1+\frac{\operatorname{var}\left(N_{+}\right)}{\left\langle N_{+}\right\rangle^{2}}+\frac{s(\omega)}{\left\langle N_{+}\right\rangle} \\
& +r[1+s(\omega)] \frac{\left(2 y_{b}-Y\right) Y-\eta^{2}}{2\left\langle N_{+}\right\rangle y_{b} Y}
\end{aligned}
$$

and for the asymmetric case

$$
\begin{gathered}
C_{p}(\eta)=1+\frac{\operatorname{var}\left(N_{A}\right)}{\left\langle N_{A}\right\rangle^{2}}+\frac{s(\omega)}{\left\langle N_{A}\right\rangle} \\
+r[1+s(\omega)] \frac{\eta\left(y_{b}-Y\right)+y_{b}\left(\eta+y_{b}\right) \log \left(\frac{Y+y_{b}}{\eta+y_{b}}\right)}{\left\langle N_{A}\right\rangle\left(\eta+y_{b}\right) Y}
\end{gathered}
$$

where $r$ is defined above Eq. (9).

\section{Appendix D: Gaussian smearing}

The application of the Gaussian smearing procedure of Eq. (19) is very simple for the considered model. In the numerator of $C\left(\eta_{1}, \eta_{2}\right)$ it amounts to replacing

$$
\left|\eta_{1}-\eta_{2}\right| \rightarrow \frac{2 \sigma_{\eta} e^{-\frac{\left(\eta_{1}-\eta_{2}\right)^{2}}{4 \sigma_{\eta}^{2}}}}{\sqrt{\pi}}+\left(\eta_{1}-\eta_{2}\right) \operatorname{erf}\left(\frac{\eta_{1}-\eta_{2}}{2 \sigma_{\eta}}\right)
$$

with the other terms $\left(\eta_{1}, \eta_{2}, \eta_{1} \eta_{2}\right)$ unchanged.
[1] G. Aad et al. (ATLAS Collaboration)(2015), ATLASCONF-2015-020

[2] G. Aad et al. (ATLAS Collaboration)(2015), ATLASCONF-2015-051
[3] S. Uhlig, I. Derado, R. Meinke, and H. Preissner, Nucl. Phys. B132, 15 (1978)K. Alpgard et al. (UA5), Phys. Lett. B123, 361 (1983)R. E. Ansorge et al. (UA5), Z. Phys. C37, 191 (1988)T. Alexopou- 
los et al. (E735 Collaboration), Phys.Lett. B353, 155 (1995)B. B. Back et al. (PHOBOS), Phys. Rev. C74, 011901 (2006)B. Abelev et al. (STAR Collaboration), Phys.Rev.Lett. 103, 172301 (2009)J. Adam et al. (ALICE), JHEP 05, 097 (2015)G. Aad et al. (ATLAS), ibid. 07, 019 (2012)S. De, T. Tarnowsky, T. K. Nayak, R. P. Scharenberg, and B. K. Srivastava, Phys.Rev. C88, 044903 (2013)

[4] A. Bzdak and D. Teaney, Phys.Rev. C87, 024906 (2013)

[5] K. Dusling, F. Gelis, T. Lappi, and R. Venugopalan, Nucl. Phys. A836, 159 (2010)A. Bzdak, Phys. Rev. C80, 024906 (2009)N. S. Amelin, N. Armesto, M. A. Braun, E. G. Ferreiro, and C. Pajares, Phys.Rev.Lett. 73, 2813 (1994)M. Braun, C. Pajares, and J. Ranft, Int.J.Mod.Phys. A14, 2689 (1999)Y.-L. Yan, D.-M. Zhou, B.-G. Dong, X.-M. Li, H.-L. Ma, et al., Phys.Rev. C81, 044914 (2010)P. Brogueira and J. Dias de Deus, Phys. Lett. B653, 202 (2007)A. Białas and K. Zalewski, Nucl.Phys. A860, 56 (2011)A. Olszewski and W. Broniowski, Phys.Rev. C88, 044913 (2013) Phys. Rev. C92, 024913 (2015)

[6] R. S. Bhalerao, J.-Y. Ollitrault, S. Pal, and D. Teaney, Phys. Rev. Lett. 114, 152301 (2015)

[7] J. Jia, S. Radhakrishnan, and M. Zhou(2015), arXiv:1506.03496 [nucl-th]

[8] A. Monnai and B. Schenke(2015), arXiv:1509.04103 [nucl-th]

[9] A. Białas, M. Błeszyński, and W. Czyż, Nucl. Phys. B111, 461 (1976)A. Białas, J. Phys. G35, 044053 (2008)

[10] A. Białas, W. Czyż, and W. Furmański, Acta Phys. Polon. B8, 585 (1977)V. V. Anisovich, Yu. M. Shabelski, and V. M. Shekhter, Nucl. Phys. B133, 477 (1978)

[11] B. Andersson, G. Gustafson, G. Ingelman, and T. Sjostrand, Phys. Rept. 97, 31 (1983)X.-N. Wang and M. Gyulassy, Phys. Rev. D44, 3501 (1991)Z.-W. Lin, C. M. Ko, B.-A. Li, B. Zhang, and S. Pal, Phys. Rev. C72, 064901 (2005)

[12] S. J. Brodsky, J. F. Gunion, and J. H. Kuhn, Phys. Rev. Lett. 39, 1120 (1977)

[13] A. Białas and M. Jeżabek, Phys. Lett. B590, 233 (2004)

[14] P. Bożek, W. Broniowski, and A. Olszewski, Phys. Rev. C92, 054913 (2015)

[15] A. Bzdak and P. Bożek(2015), arXiv:1509.02967 [hep-ph]

[16] L.-G. Pang, H. Petersen, G.-Y. Qin, V. Roy, and X.-N. Wang(2015), arXiv:1511.04131 [nucl-th]

[17] V. Khachatryan et al. (CMS), Phys. Rev. C92, 034911 (2015)
[18] P. Bożek, W. Broniowski, and J. Moreira, Phys. Rev. C83, 034911 (2011)

[19] H. Petersen, V. Bhattacharya, S. A. Bass, and C. Greiner, Phys.Rev. C84, 054908 (2011)

[20] K. Xiao, F. Liu, and F. Wang, Phys.Rev. C87, 011901 (2013)

[21] L.-G. Pang, G.-Y. Qin, V. Roy, X.-N. Wang, and G.-L. Ma, Phys. Rev. C91, 044904 (2015)

[22] J. Jia and P. Huo, Phys.Rev. C90, 034915 (2014)

[23] P. Bożek, W. Broniowski, and A. Olszewski, Phys.Rev. C91, 054912 (2015)

[24] P. Bożek and W. Broniowski(2015), arXiv:1506.02817 [nucl-th]

[25] W. Czyż and L. C. Maximon, Annals Phys. 52, 59 (1969)

[26] M. L. Miller, K. Reygers, S. J. Sanders, and P. Steinberg, Ann. Rev. Nucl. Part. Sci. 57, 205 (2007)

[27] A. Białas, K. Fiałkowski, W. Słomiński, and M. Zieliński, Acta Phys. Polon. B8, 855 (1977)

[28] A. Białas and W. Czyż, Acta Phys. Polon. B10, 831 (1979)

[29] A. Białas and A. Bzdak, Phys. Lett. B649, 263 (2007)Phys. Rev. C77, 034908 (2008)

[30] M. Miller and R. Snellings(2003), nucl-ex/0312008

[31] J. D. Bjorken, Intl. Summer Inst. in Theoretical Physics on Current Induced Reactions Hamburg, Germany, September 15-26, 1975, Lect. Notes Phys. 56, 93 (1976)

[32] R. Ryblewski and W. Florkowski, J. Phys. G38, 015104 (2011)

[33] M. Martinez and M. Strickland, Nucl. Phys. A848, 183 (2010)

[34] A. Białas and W. Czyż, Acta Phys. Polon. B36, 905 (2005)

[35] P. Bożek and W. Broniowski, Phys. Rev. C88, 014903 (2013)

[36] W. Broniowski, M. Rybczyński, and P. Bożek, Comput. Phys. Commun. 180, 69 (2009)M. Rybczyński, G. Stefanek, W. Broniowski, and P. Bożek, 185, 1759 (2014)

[37] S. Eremin and S. Voloshin, Phys. Rev. C67, 064905 (2003)

[38] S. S. Adler et al. (PHENIX), Phys. Rev. C89, 044905 (2014)

[39] J. Adam et al. (ALICE)(2015), arXiv:1509.07255 [nucl$\mathrm{ex}]$

[40] S. Jeon and S. Pratt, Phys. Rev. C65, 044902 (2002)P. Bożek and W. Broniowski, Phys. Rev. Lett. 109, 062301 (2012)

[41] P. Bożek, Phys. Rev. C85, 034901 (2012)

[42] A. Adil, M. Gyulassy, and T. Hirano, Phys. Rev. D73, 074006 (2006)P. Bożek and I. Wyskiel, C81, 054902 (2010) 